\title{
Unconstrained Shoulder Joint Position Sense Does Not Change with Body Orientation
}

\author{
Jason Chapman, ${ }^{3}$ David N. Suprak, ${ }^{2}$ Andrew R. Karduna ${ }^{1}$ \\ ${ }^{1}$ Department of Human Physiology, College of Arts and Sciences, University of Oregon, Eugene, Oregon 97403-1240, ${ }^{2}$ Department of Physical \\ Education, Western Washington University, Bellingham, Washington 98225, ${ }^{3}$ Department of Health, Physical Education and Dance, Emory \\ University, Atlanta, Georgia 30322
}

Received 13 May 2008; accepted 20 October 2008

Published online in Wiley InterScience (www.interscience.wiley.com). DOI 10.1002 /jor.20813

\begin{abstract}
Our knowledge of the role of muscle activation on proprioception is incomplete. Previous work has either focused on comparing active and passive motions or manipulated both muscle activation and joint angles simultaneously. We conducted an experiment at the shoulder in which subjects' trunks were tilted backwards to decouple joint angle from joint torque. Twenty three healthy subjects underwent testing in an unconstrained joint position sense task. Kinematics were measured with a magnetic tracking device. The joint position sense task consisted of subjects moving their arms to a predetermined orientation in space with the help of visual feedback from the magnetic tracking device presented to the subjects through a head-mounted display. Subjects were then asked to reproduce the presented joint position in the absence of visual feedback. The protocol was performed under two tilts: upright and trunk tilted back $45^{\circ}$. This allowed for a comparison of joint position sense at different joint angles (at the same resistive torque) and at different resistive torques (at the same joint angles). When comparing these two tilts, we found that matching based on elevation angle demonstrated no significant difference, while matching based on torques did find differences. These results implicate elevation angle at the shoulder as playing a more important role in modulating joint position sense than joint torque.@ 2008 Orthopaedic Research Society. Published by Wiley Periodicals, Inc. J Orthop Res
\end{abstract}

Keywords: proprioception; joint position sense; shoulder; muscle activation

Muscle forces at the shoulder play a predominant role in maintaining joint stability during motion. ${ }^{1,2}$ Muscle activation is partly regulated through a feedback mechanism receiving information from muscle and joint receptors and consequently producing a variation of a specific motor program. ${ }^{3}$ These receptors were identified by Sherrington ${ }^{4}$ as the proprioceptive-field and defined as the field in which receptors are stimulated by the organism itself. Sherrington went on to describe how an external stimulus causes an action to occur, and this action stimulates the proprioceptors, which can initiate their own reflex. This work paved the way for research involving proprioception, the term used to describe "afferent information arising from internal peripheral areas of the body that contribute to postural control, joint stability, and several conscious sensations."

The afferent information from joint receptors, along with signals from the muscle spindles and Golgi tendon organs, account for joint position sense (JPS), or the ability of an individual to know where a limb is located in space. ${ }^{3}$ JPS consists of two parts: static position sense, or the perception of a stationary joint angle, and dynamic position sense, or the ability to identify the position of a joint during a movement. ${ }^{5,6}$ In contrast, kinesthesia is defined as the ability to sense movement and is typically assessed as the detection of joint motion. ${ }^{7}$

Evidence exists in the literature that an increase in muscle activation results in an improvement in joint proprioception. This relation has been demonstrated by comparing active and passive motion for the

Correspondence to: Andrew R. Karduna (T: 541-346-0438; F: 541346-2841; E-mail: karduna@uoregon.edu)

(c) 2008 Orthopaedic Research Society. Published by Wiley Periodicals, Inc. knee,${ }^{8,9}$ elbow, ${ }^{10,11}$ wrist, ${ }^{12}$ finger, ${ }^{11}$ and shoulder. ${ }^{11,13,14}$ Improved proprioception leads to an increase in reflex activity and may enhance joint stability through co-activation of the musculature surrounding a joint. ${ }^{15}$ This effect is especially important at the shoulder, where the ligaments and bony structures offer somewhat less stability than at other joints. Minimal passive support coupled with a large range of motion at the shoulder may result in a greater need for accurate proprioception to maintain stability as compared to other joints. A better understanding of the role played by muscle activation in JPS may help refine rehabilitation techniques aimed at improving joint proprioception after injury. ${ }^{16,17}$

A previous study from our laboratory appears to be the first to demonstrate a graded response in JPS at different levels of muscle activation. ${ }^{18}$ In that study, an improvement in accuracy during an unconstrained active JPS task was observed with an increase in muscle activation (as gauged by an increase in resistive torque due to gravity). However, a limitation of the study was that changes in muscle activation were accompanied by changes in joint angle since gravity provided the external resistance. Therefore, the changes in JPS could have been due to either factor. The present study was designed to overcome this limitation. By manipulating the trunk angle, we propose a model in which we can directly compare JPS at different joint angles (at the same resistive torque) and at different resistive torques (at the same joint angles). Previous work in our lab demonstrated an effect of elevation angle (where torque is changing) and no effect of plane of elevation (where torque is not changing) on JPS accuracy. We therefore hypothesized that JPS accuracy is primarily affected by changes in resistive torque, rather than by changes in joint angle. 


\section{METHODS}

\section{Subjects}

Twenty-two healthy individuals (13 males, 10 females; mean age $=21.7$ years \pm 4.8 ; mean body $\operatorname{mass}=70.6 \pm 10.4 \mathrm{~kg}$ ) agreed to participate. In general, subjects were healthy college students. Prior to testing, all subjects signed an informed consent form approved by the university's Institutional Review Board. Subjects were excluded if they had any history of shoulder injury or surgery. No subject had any known neurological condition that might have adversely affected proprioception.

\section{Instrumentation}

Kinematic data were collected with a magnetic tracking device (Fastrak, Polhemus, Colchester, VT). The Polhemus unit consists of a transmitter, three receivers, and a digitizer. The transmitter emits an electromagnetic field that is sensed by the receivers and digitizer. The strength and orientation of these signals are used to determine the relative position and orientation of the receivers in space. To track the movement of the humerus with respect to the thorax during testing, receivers were placed on the sternum, about $1.5 \mathrm{~cm}$ inferior to the jugular notch ${ }^{19}$ and on the humerus just above the lateral epicondyle via a custom-molded cuff made from splinting material (Polyform, Sammons Preston Rolyan, Bolingbrook, IL). Additionally, a receiver was fastened to the scapula with a previously validated tracking jig $^{20}$ for digitization purposes, but was removed prior to testing. Following attachment of the receivers, bony landmarks were digitized on the thorax and humerus to establish anatomical coordinate systems in accordance with the standard proposed by the International Society of Biomechanics. ${ }^{21}$ Euler angles were used to represent sequence-dependant humeral rotations with respect to the thorax, consisting of the plane of elevation and degree of elevation, as described by An et al. ${ }^{22}$

Proprioception was tested at the shoulder while minimizing other sensory input. To occlude visual clues, each subject wore a head-mounted display (i-glasses, I-O Display Systems, Sacramento, CA). The unit was retrofitted with felt attached to the top, bottom, and sides, which eliminated external light sources. The display allowed for presentation of kinematic output from the computer to the subject during testing while suppressing extraneous visual cues related to the position and movement of the shoulder joint (Fig. 1).

A chair was designed to allow the torso to be tilted backward from the upright sitting position to a reclined position of $45^{\circ}$ (Fig. 1). The chair provided support at the lower back and along the spine, but minimized cutaneous input to the scapula and shoulder region. The chair was also outfitted with an adjustable headrest so the subject received similar cutaneous input while upright and in the tilted position. Cutaneous input was present across the lower back and up the spine. While the activation of the neck muscles might have differed between the two tilts, we utilized a headrest to support the head while the subjects were tilted back, so no excess muscle activation was necessary to counter-balance the torque due to the weight of the head.

\section{Protocol}

All testing was performed on the dominant upper extremity and in a single session. Since "warming up" before exercise has been shown to affect proprioception, ${ }^{23}$ each subject completed a standardized warm-up prior to testing to ensure a constant starting point for all subjects. ${ }^{18}$ This consisted of Codman's pendulum exercises (rotations and sagittal plane motion)
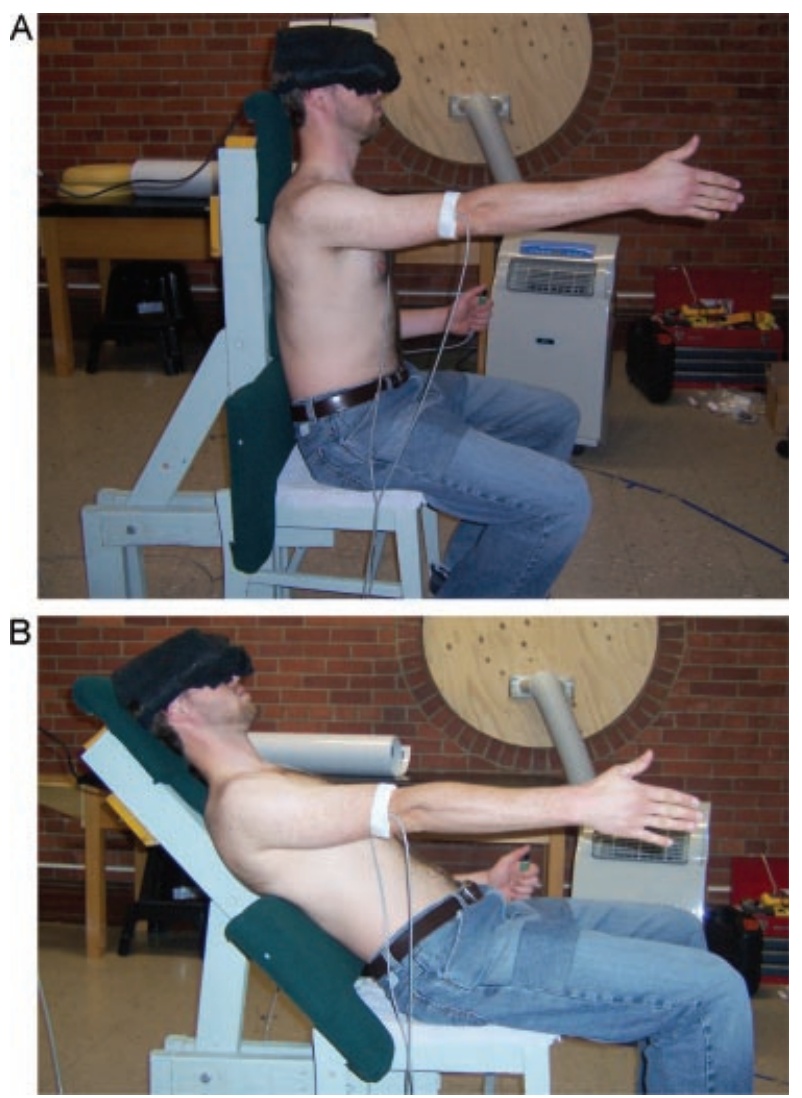

Figure 1. Demonstration of the two experimental tilts in which the target was $90^{\circ}$ of humeral elevation with respect to gravity for the (A) upright and (B) tilted back $45^{\circ}$

followed by stretches consisting of holding a static external and then internal rotation position, both with the shoulder abducted to approximately $90^{\circ}$, for two sets of $15 \mathrm{~s}$ each. Upon completion of the warm-up, subjects removed their shirts (females wore sports bras) and any jewelry or accessories that could affect JPS through tactile cues during testing. Bony landmarks were digitized, and then subjects were transferred to the test-specific chair. Subjects were thoroughly instructed as to the testing protocol, and practice trials were completed until the subject felt comfortable with the protocol. Subjects were then fitted with the head-mounted display and further practice trials were completed so that subjects would become comfortable with its use.

The initial chair position was randomly chosen as upright or tilted. Seven target positions were presented to the subjects in a randomized order according to a balanced Latin square design, ${ }^{24}$ with $15 \mathrm{~s}$ separating each trial. After successfully completing all seven trials, subjects were given a 1-min break, and then the same seven positions were repeated in a different randomized order. The subjects were then given a 5-min break during which time the chair was adjusted to the other position (upright or tilted). Testing at the same seven target positions (twice) was then performed at the new tilting angle.

Each trial began with the subject's dominant arm at the side resting against the chair, and all arm motion was accomplished with the elbow in full extension. The nondominant arm was either at the subject's side or in the subject's lap, depending on individual comfort. In the head-mounted display, the subject was presented with the output from custom-written software (Labview, National Instruments, Austin, TX), which served to 
guide and instruct the subject throughout the protocol. A graycolored screen with a black outlined box in the center appeared first. At one side was a vertical red bar, and a horizontal red bar was present at the top or bottom. The red bars were presented to guide the subject's arm movement toward the box at the center of the screen. More specifically, a red bar on the right side indicated the subject must move the arm toward the right, a red bar at the top indicated a necessary move toward the top, etc. When the subject's shoulder position varied no more than $5^{\circ}$ from the target position in both plane and elevation angles, the red bars disappeared and were replaced with a red dot on the screen depicting the current shoulder position. The subject then continued to guide the arm to the appropriate target plane and elevation angle, which was achieved by moving the red dot into the box. Once this was accomplished, the entire screen went blank, and the subject was required to maintain the position for $5 \mathrm{~s}$ while focusing on the shoulder joint. Then a voice command initiated from the program instructed the subject to "relax," at which time the subject returned the arm to the starting position. After $3 \mathrm{~s}$ and with the screen remaining blank, the subject was instructed to "return" the arm back to the position that was previously presented. When the subject believed that the arm and shoulder were in the same position, the subject depressed a trigger button held in the contralateral hand. An audible "beep" then sounded, and the subject returned to the starting position.

The target positions consisted of $30,45,60,75,90,105$, and $120^{\circ}$ of humerothoracic elevation. The selection of target angles allowed for comparisons of the effect of torque and angle at different tilts. Since tilting occurred in the sagittal plane, ideally, the plane of elevation should have also occurred in this plane. However, to ensure minimal contact with any part of the subjects' thighs, all targets occurred in a plane that was $10^{\circ}$ lateral to the sagittal plane (i.e., to the right for righthanded subjects and to the left for left-handed subjects). When the trunk was tilted backwards, calculations indicated an insignificant difference in external shoulder torque $(\sim 1 \%)$ between arm elevation in the sagittal plane compared to the tested plane.

\section{Data Analysis}

Kinematic data were converted into humeral plane and elevation angles using transformation matrices between the coordinate systems of the thorax and humerus. These plane and elevation angles were then converted into a $3 \mathrm{D}$ vector originating at the center of the humeral head and ending at the midpoint between the medial and lateral humeral epicondyles. The angle between the presented and reproduced vector represented the magnitude of the repositioning error. ${ }^{18}$

Statistical analyses were performed using SPSS, version 15 (SPSS, Chicago, IL). To determine the significance of tilt on repositioning error scores, a two-way, repeated measures ANOVA was conducted with two within-subject factors (tilt and target angles). Due to the nature of the assigned target angles, four target positions for each tilt were matched so as to require the same torque (humeral angle with respect to gravity) at the shoulder to achieve that position at each tilt (Table 1). An additional two-way repeated measures ANOVA was performed with two within-subject factors (tilt and joint torque). This analysis was performed to determine whether a significant difference in error scores was present between target angles that corresponded to the same torque at different tilts and different elevation angles. For both analyses, if there was a significant effect of the tilt angle and a significant
Table 1. Target Elevation Angles for Upright and Tilted Trials $^{a}$

\begin{tabular}{lcr}
\hline & \multicolumn{2}{c}{$\begin{array}{c}\text { Humerus with Respect to } \\
\text { Thorax (degrees) }\end{array}$} \\
\cline { 2 - 3 } $\begin{array}{l}\text { Humerus with Respect } \\
\text { to Gravity (degrees) }\end{array}$ & Upright & Tilted \\
\hline 30 & 30 & \\
45 & 45 & \\
60 & 60 & 30 \\
75 & 75 & 45 \\
90 & 90 & 60 \\
105 & 105 & 75 \\
120 & 120 & 90 \\
135 & & 105 \\
150 & & 120 \\
165 & & \\
\hline
\end{tabular}

${ }^{a}$ Note the angles that match based on the elevation angle of the humerus with respect to the gravity.

interaction between the two factors, follow-up paired $t$-tests were run. The alpha level was set at 0.05 . However, to account for multiple comparisons, a Bonferroni-Holm correction was utilized.

\section{RESULTS}

Despite our efforts to avoid the arm bumping into the thigh, about half of the trials at $30^{\circ}$ of elevation in the upright tilt resulted in contact. Therefore, only data from the remaining six angles were included. The twoway ANOVA with matching joint angles revealed no main effect of tilt on the magnitude of the repositioning error $(p=0.494)$ and a significant interaction between tilt and joint angle $(p<0.05)$ (Fig. 2A). Follow-up paired $t$-tests demonstrated that for all joint angles, no significant difference existed between tilts $(p>0.05)$. The two-way ANOVA with matching joint torques revealed a main effect of tilt on the magnitude of the repositioning error $(p=0.002)$ and no significant interaction between tilt and joint angle $(p=0.970)$ (Fig. $2 \mathrm{~B})$.

\section{DISCUSSION}

Shoulder proprioception testing has traditionally been performed under constrained and passive conditions, but recent unconstrained active repositioning models have appeared in the literature. ${ }^{25,26}$ Here we revisited our own developed JPS model ${ }^{18}$ to further analyze the effect of torque and joint angle on repositioning errors. The tilting chair allowed for comparison of repositioning error at the same elevation angles reached by implementing different levels of torque and the error associated with different elevation angles but equal torque (Table 1).

Our goal was to determine whether the increase in JPS accuracy previously noted with increasing humeral elevation $^{18}$ was due to changing joint angles or joint torques. Results demonstrated no significant difference when the two tilts were compared on the basis of joint angle, but a significant effect occurred when they were 

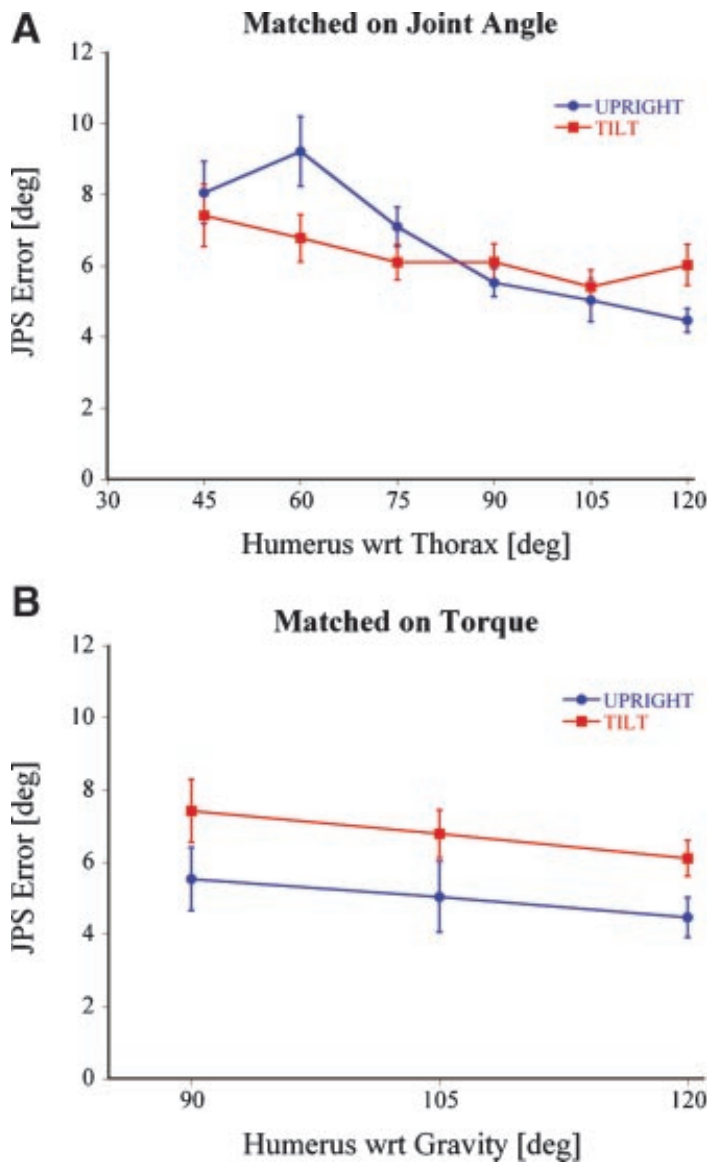

Figure 2. Vector error mean ( \pm SEM) for upright and tilted trials, matched based on (A) joint angles and (B) torque.

matched on joint torque. In a parallel study, we found that by increasing joint torque with the addition of weights, an increase in JPS accuracy occurred. ${ }^{27}$ However, the magnitude of that effect was much smaller than what we observed in our elevation study. ${ }^{18}$ One theory that would fit the data from all three experiments is that effects exist for both joint angle (present study) and joint torque ${ }^{27}$ but the effect of angle is much stronger. In the present study, for five of the six angles (Fig. 2A), the tilt with the higher joint torque exhibited a low mean error (tilting at 45 and $60^{\circ}$ and upright at 90,105 , and $120^{\circ}$ ). Testing more subjects might have resulted in significant differences due to tilting of the trunk.

The magnitude of the difference observed in the current study is about $2^{\circ}$ (Fig. 2B). The clinical relevance of this small difference is debatable, but it represents an error increase of about $35 \%$. Also, the magnitude is similar to changes in shoulder proprioception due to fatigue. ${ }^{28-30}$

It is likely that humans incorporate both muscle activation (related to torque) and some intrinsic coordinate system based on muscle length, joint receptor activation, and skin deformation. Studies from our lab ${ }^{18}$ and others ${ }^{31-33}$ have been presented supporting the muscle activation model. Conversely, Darling and Miller $^{34}$ reported that subjects replicated arm positions with respect to the trunk with more accuracy than arm positions with respect to gravity, and concluded that perhaps some intrinsic coordinate system exists that the brain can integrate when visual cues are absent. That the body may preferentially use one input more than another, or in combination with another, as opposed to selecting which afferent system to utilize at a given time seems intuitive. Our study shows that shoulder JPS is maintained even when visual cues are absent and the vestibular input is altered.

Darling and Miller ${ }^{34}$ also manipulated trunk orientation to change the relationship between joint angles and torques at the shoulder. However trunk orientation was changed between the positioning and repositioning motions. Similarly, other investigators manipulated the orientation of the arm to change the relationship between joint angles and torques at the elbow. ${ }^{35-39}$ However, in all these studies, humeral orientation was different between the positioning and matching motions (regardless of whether a bilateral or unilateral experiment was performed). To our knowledge, only Gooey et al. ${ }^{40}$ manipulated the arm angle between trials, and they found a decrease in the magnitude, but an increase in the variability of errors when the arm was elevated. However, the effect of gravity is difficult to determine as the presented angles were not reported.

For upright tilting in the present study, a general trend of decreasing errors occurred as joint angle increased, consistent with other studies that found JPS is heightened at the end range of motion. ${ }^{41-43}$ However, we found the highest accuracy at $90^{\circ}$ of elevation. This difference could be attributed to several factors. The subjects in our study performed the repositioning task while seated in a chair that provided head and back support. The effect of torso and head support may be insignificant, but the presence of tactile cues increases perception of movement ${ }^{44}$ and may override joint receptors in motion detection. ${ }^{45}$ This cutaneous input was curtailed by providing only enough skin contact to support the torso and head adequately while leaving the scapula and surrounding areas free to move without cutaneous feedback. Nonetheless, skin stretched at a large distance from a joint still elicits a sensation of movement at that joint. ${ }^{46}$ Elevation close to the sagittal plane (as opposed to the scapular plane in our previous work) may have caused a larger stretch on the posterior capsular tissues resulting in increased anterior translation. ${ }^{47}$ Both effects could have served to increase afferent feedback from the shoulder.

A primary assumption in our model, that similar resistive torques at different arm positions correspond to similar muscle activation patterns, is overly simplistic. We are essentially using resistive torque as a surrogate measurement of muscle force. Other factors argue against this assumption, such as differences in clavicular and scapular kinematics, ${ }^{48,49}$ muscle moment arms, ${ }^{50,51}$ and inter-muscle recruitment patterns. ${ }^{52,53}$ However, we believe that our study represents the next step towards understanding mechanisms that control 
shoulder JPS. For the present study, motions were performed with no external load on the arm (other than the resistive torque due to the weight of the upper extremity). This is common for JPS studies, but caution should be taken when applying these results to larger resistive loads.

The vestibular input in our study is altered in that the visual input of body orientation is occluded, but the subjects do receive visual input as to the relation of their arm to the target on the computer screen. In this situation, the vestibular and visual inputs are not in conflict, so we would argue that the vestibular system normalizes the body sense to the tilted position during those trials in which the subjects are tilted. ${ }^{54}$ Furthermore, Paloski, et al. ${ }^{55}$ reported "that healthy subjects can adequately compensate for different head orientations with respect to gravity by maintaining postural stability during static and low frequency dynamic head tilts, in the absence of vision and accurate somatosensory inputs." Another study concludes that whole-body proprioception is integrated with the vestibular system to generate a reference of self-motion in the absence of visual feedback, which supports the argument that an alteration in head position does not change proprioceptive feedback at the shoulder. ${ }^{56}$ Additionally, the anatomy of the vestibular system is defined as sensing linear and rotational accelerations, but when the head is stationary, the system adapts to gravity and references the body accordingly. ${ }^{57}$

In summary, when comparing upright and tilted back $45^{\circ}$, matching based on shoulder elevation angle demonstrated no significant difference, while matching based on torques did find differences. This result would appear to implicate elevation angle as more important in joint position sense than joint torque, but we cannot rule out an effect of torque that was overshadowed in the present study. The results of our work may lend insight into the understanding of the mechanisms underlying joint stability maintenance and may have important implications for rehabilitation. Improved shoulder JPS under conditions of either increased muscle activation or joint angles may represent a strategy for avoiding injury and maintaining coordinated movement patterns while performing functional activities involving considerable muscular demands, such as those involving high forces, since these are the activities in which joint injuries are most likely to occur. The extent to which we can better understand how JPS improves under conditions of increased external torque may help guide clinicians in selecting functional rehabilitation exercises with external loads or in positions of increased external torque to promote joint stability during movement.

\section{REFERENCES}

1. Malicky DM, Soslowsky LJ, Blasier RB, et al. 1996. Anterior glenohumeral stabilization factors: progressive effects in a biomechanical model. J Orthop Res 14:282288.
2. Lee SB, Kim KJ, O'Driscoll SW, et al. 2000. Dynamic glenohumeral stability provided by the rotator cuff muscles in the mid-range and end-range of motion. A study in cadavera. J Bone Joint Surg [Am] 82:849-857.

3. Riemann BL, Lephart SM. 2002. The sensorimotor system, part II: the role of proprioception in motor control and functional joint stability. J Athl Train 37:80-84.

4. Sherrington C. 1906. The integrative action of the nervous system. New Haven, CT: Yale University Press.

5. Clark FJ, Burgess RC, Chapin JW. 1986. Proprioception with the proximal interphalangeal joint of the index finger. Evidence for a movement sense without a static-position sense. Brain 109(Pt 6):1195-1208.

6. Bevan L, Cordo P, Carlton L, et al. 1994. Proprioceptive coordination of movement sequences: discrimination of joint angle versus angular distance. J Neurophysiol 71: 1862-1872.

7. Chieffi S, Conson M, Carlomagno S. 2004. Movement velocity effects on kinaesthetic localisation of spatial positions. Exp Brain Res 158:421-426.

8. Bullock-Saxton JE, Wong WJ, Hogan N. 2001. The influence of age on weight-bearing joint reposition sense of the knee. Exp Brain Res 136:400-406.

9. Stillman BC, McMeeken JM. 2001. The role of weightbearing in the clinical assessment of knee joint position sense. Aust J Physiother 47:247-253.

10. Taylor JL, McCloskey DI. 1992. Detection of slow movements imposed at the elbow during active flexion in man. J Physiol 457:503-513.

11. Hall LA, McCloskey DI. 1983. Detections of movements imposed on finger, elbow and shoulder joints. J Physiol 335: $519-533$

12. Colebatch JG, McCloskey DI. 1987. Maintenance of constant arm position or force: reflex and volitional components in man. J Physiol 386:247-261.

13. Laufer Y, Hocherman S, Dickstein R. 2001. Accuracy of reproducing hand position when using active compared with passive movement. Physiother Res Int 6:65-75.

14. Brindle TJ, Uhl TL, Nitz AJ, et al. 2006. The influence of external loads on movement precision during active shoulder internal rotation movements as measured by 3 indices of accuracy. J Athl Train 41:60-66.

15. Allum JH, Honegger F, Pfaltz CR. 1989. The role of stretch and vestibulo-spinal reflexes in the generation of human equilibrating reactions. Prog Brain Res 80:399-409.

16. Lephart SM, Pincivero DM, Giraldo JL, et al. 1997. The role of proprioception in the management and rehabilitation of athletic injuries. Am J Sports Med 25:130-137.

17. Wilk KE, Meister K, Andrews JR. 2002. Current concepts in the rehabilitation of the overhead throwing athlete. Am J Sports Med 30:136-151.

18. Suprak DN, Osternig LR, van Donkelaar P, et al. 2006. Shoulder joint position sense improves with elevation angle in a novel, unconstrained task. J Orthop Res 24:559-568.

19. Borstad JD, Ludewig PM. 2002. Comparison of scapular kinematics between elevation and lowering of the arm in the scapular plane. Clin Biomech (Bristol, Avon) 17:650-659.

20. Karduna AR, McClure PW, Michener LA, et al. 2001. Dynamic measurements of three-dimensional scapular kinematics: a validation study. J Biomech Eng 123:184-190.

21. Wu G, van der Helm FC, Veeger HE, et al. 2005. ISB recommendation on definitions of joint coordinate systems of various joints for the reporting of human joint motion-part II: shoulder, elbow, wrist and hand. J Biomech 38:981-992.

22. An KN, Browne AO, Korinek S, et al. 1991. Three-dimensional kinematics of glenohumeral elevation. J Orthop Res 9:143149 . 
23. Bartlett MJ, Warren PJ. 2002. Effect of warming up on knee proprioception before sporting activity. Br J Sports Med 36: 132-134.

24. Portney LG, Watkins MP. 2000. Foundations of clinical research: application to practice. Upper Saddle River, NJ: Prentice Hall Health.

25. Barden JM, Balyk R, Raso VJ, et al. 2004. Dynamic upper limb proprioception in multidirectional shoulder instability. Clin Orthop 420:181-189.

26. Tripp BL, Uhl TL, Mattacola CG, et al. 2006. A comparison of individual joint contributions to multijoint position reproduction acuity in overhead-throwing athletes. Clin Biomech (Bristol, Avon) 21:466-473.

27. Suprak DN, Osternig LR, van Donkelaar P, et al. 2007. Shoulder joint position sense improves with external load. J Mot Behav 39:517-525.

28. Voight ML, Hardin JA, Blackburn TA, et al. 1996. The effects of muscle fatigue on and the relationship of arm dominance to shoulder proprioception. J Orthop Sports Phys Ther 23:348352.

29. Lee HM, Liau JJ, Cheng CK, et al. 2003. Evaluation of shoulder proprioception following muscle fatigue. Clin Biomech (Bristol, Avon) 18:843-847.

30. Carpenter JE, Blasier RB, Pellizzon GG. 1998. The effects of muscle fatigue on shoulder joint position sense. Am J Sports Med 26:262-265.

31. Swanik KA, Lephart SM, Swanik CB, et al. 2002. The effects of shoulder plyometric training on proprioception and selected muscle performance characteristics. J Shoulder Elbow Surg 11:579-586.

32. Myers JB, Lephart SM. 2000. The role of the sensorimotor system in the athletic shoulder. J Athl Train 35:351363.

33. Mercier C, Bertrand AM, Bourbonnais D. 2004. Differences in the magnitude and direction of forces during a submaximal matching task in hemiparetic subjects. Exp Brain Res 157: $32-42$.

34. Darling WG, Miller GF. 1995. Perception of arm orientation in three-dimensional space. Exp Brain Res 102:495502.

35. Bock O. 1994. Joint position sense in simulated changedgravity environments. Aviat Space Environ Med 65:621626 .

36. Worringham CJ, Stelmach GE. 1985. The contribution of gravitational torques to limb position sense. Exp Brain Res 61: $38-42$.

37. Worringham CJ, Stelmach GE, Martin ZE. 1987. Limb segment inclination sense in proprioception. Exp Brain Res 66:653-658.

38. Darling WG. 1991. Perception of forearm angles in 3-dimensional space. Exp Brain Res 87:445-456.

39. Garrett SR, Pagano C, Austin G, et al. 1998. Spatial and physical frames of reference in positioning a limb. Percept Psychophys 60:1206-1215.

40. Gooey K, Bradfield O, Talbot J, et al. 2000. Effects of body orientation, load and vibration on sensing position and movement at the human elbow joint. Exp Brain Res 133: $340-348$

41. Janwantanakul P, Magarey ME, Jones MA, et al. 2001. Variation in shoulder position sense at mid and extreme range of motion. Arch Phys Med Rehabil 82:840-844.

42. Lonn J, Crenshaw AG, Djupsjobacka M, et al. 2000. Position sense testing: influence of starting position and type of displacement. Arch Phys Med Rehabil 81:592-597.

43. Grigg P, Hoffman AH. 1989. Calibrating joint capsule mechanoreceptors as in vivo soft tissue load cells. J Biomech 22:781-785

44. Collins DF, Refshauge KM, Todd G, et al. 2005. Cutaneous receptors contribute to kinesthesia at the index finger, elbow, and knee. J Neurophysiol 94:1699-1706.

45. Edin BB, Johansson N. 1995. Skin strain patterns provide kinaesthetic information to the human central nervous system. J Physiol 487(Pt 1):243-251.

46. Burgess PR, Wei JY, Clark FJ, et al. 1982. Signaling of kinesthetic information by peripheral sensory receptors. Annu Rev Neurosci 5:171-187.

47. Harryman DT II, Sidles JA, Clark JM, et al. 1990. Translation of the humeral head on the glenoid with passive glenohumeral motion. J Bone Joint Surg [Am] 72A:1334-1343.

48. McClure PW, Michener LA, Sennett BJ, et al. 2001. Direct 3dimensional measurement of scapular kinematics during dynamic movements in vivo. J Shoulder Elbow Surg 10: 269-277.

49. Ludewig PM, Cook TM. 2000. Alterations in shoulder kinematics and associated muscle activity in people with symptoms of shoulder impingement. Phys Ther 80:276-291.

50. Liu J, Hughes RE, Smutz WP, et al. 1997. Roles of deltoid and rotator cuff muscles in shoulder elevation. Clin Biomech (Bristol, Avon) 12:32-38.

51. Graichen H, Englmeier KH, Reiser M, et al. 2001. An in vivo technique for determining 3D muscular moment arms in different joint positions and during muscular activationapplication to the supraspinatus. Clin Biomech (Bristol, Avon) 16:389-394.

52. Gribble PL, Mullin LI, Cothros N, et al. 2003. Role of cocontraction in arm movement accuracy. J Neurophysiol 89:2396-2405.

53. d'Avella A, Portone A, Fernandez L, et al. 2006. Control of fast-reaching movements by muscle synergy combinations. J Neurosci 26:7791-7810.

54. Orlov IV, Stolbkov YK, Shuplyakov VS. 2006. Vestibular influences on postural instability induced by movements of the visual environment and support. Neurosci Behav Physiol 36:297-305.

55. Paloski WH, Wood SJ, Feiveson AH, et al. 2006. Destabilization of human balance control by static and dynamic head tilts. Gait Posture 23:315-323.

56. Schweigart G, Chien RD, Mergner T. 2002. Neck proprioception compensates for age-related deterioration of vestibular self-motion perception. Exp Brain Res 147:89-97.

57. Kandel E, Schwartz J, Jessell T. 2000. Principles of neural science. New York: McGraw-Hill. 